\title{
Influence du poids de la semence et de la profondeur de semis sur la croissance et le développement de la plantule de maïs
}

\author{
B Pommel \\ avec la collaboration technique de $\mathrm{C}$ Bouchard \\ INRA, Laboratoire d'agronomie, 78850 Thiverval-Grignon, France
}

(Reçu le 27 décembre 1989; accepté le 7 septembre 1990)

\begin{abstract}
Résumé - On a étudié, en conditions semi-contrôlées sous serre, la croissance et le développement de plantules de maîs selon le poids de la semence, la profondeur de semis et la variété (4). Pendant la phase hétérotrophe, la croissance de l'hydride DEA est, à peu de choses près, directement proportionnelle à la quantité de réserves de la graine utilisées depuis la germination, dans un rapport peu variable suivant les conditions (cœefficient de variation de $3,1 \%$ ). Les plantules issues de grosses semences présentent une croissance nettement plus rapide, mais une efficience légèrement moindre pour l'utilisation des réserves de la graine.

Les stades foliaires ou racinaires sont déterminés par l'âge de la plantule depuis sa germination, indépendamment de la taille de la semence d'origine, et, après l'apparition de la $2^{\theta}$ feuille, indépendamment de la profondeur de semis.

Les plantules issues de semis profonds utilisent un peu plus rapidement les réserves de la semence et montrent un taux de croissance supérieur.

La fraction des réserves mobilisées jusqu'au passage à l'autotrophie est peu variable, autour de $65 \%$ : les plantules issues de grosses semences débutent donc leur période photosynthétique avec une plus grande quantité de réserves encore disponibles dans la semence et avec un poids supérieur d'organes chlorophylliens.
\end{abstract}

maïs / poids de la semence / profondeur de semis / utilisation des réserves de la semence / taux de croissance

Summary - Effects of seed weight and sowing depth on growth and development of maize seedlings. Growth and development of maize seedlings according to seed weight, depth of sowing and variety (4) were studied in a greenhouse. Up to $240^{\circ}$ per d, the growth of the DEA variety is directly proportional to the amount of seed reserves which have been used since germination: the ratio shows little variation according to seed weight and sowing depth (the variation cœefficient is $3.1 \%$, figs 1 and 2).

The slight advantage of seedlings originating from light seeds in relation to the relative amount of reserves which have been utilized during a given period of time (table I) is quite insufficient to counterbalance the much larger absolute amount used from heavy seeds. As a consequence, the seedlings originating from heavy seeds displayed rapid growth (fig 3).

We found a genetical variation in the rhythm of seed reserves utilisation as follows: Monarque was more rapid, followed by $D E A, F_{7} \times F_{2}$ then Mohican (table $V$ ). These differences have consequences on the rate of growth during the heterotrophic stage, which is higher with Monarque.

Unlike the growth, the development (appearance of new organs as leaves or ramifications on the radicle or lateral roots) is independent of the weight of the originating seed. It expresses the physiological age of the seedling, according to the time passed, as the sum of temperature (table II).

The ratio of reserves used when a seedling starts an autotrophic life is about $65 \%$, showing little variation. At that time, seedlings have passed the 3rd leaf stage, the advent of ramifications on lateral roots, and we observe a temporary decrease in root weight as described by Bourdu and Gregory (1983) (table III).

On the deep sowing treatments, the seedlings use seed reserves more rapidly and as a consequence of this, grow faster (table $\mathrm{N}$ ). In these situations the emergence is comparatively delayed with shallow sowings, but this difference of stage disappears after the advent of the 1st leaf.

When the ermergence takes place, the seedlings originating from light seeds sowed deeply dispose of only $45 \%$ of maternal reserves (ie $75 \mathrm{mg}$ ) in contrast with those from heavy seeds and shallow sowing which still dispose of $71 \%$ (1e $235 \mathrm{mg}$ ). In the first situation, the safety margins, in the event of adverse conditions (cold weather, excess of water, coarse seed-bed, surface crust) are restricted. On the other hand, the weight of chlorophyllian organs, when efficient photosynthesis takes place, is much higher in seedlings originating from heavy seeds rather than from light seeds: the initial state of the exponential growth is much better, and, as a consequence, this fact could induce better progress at all stages. 


\section{INTRODUCTION}

La structure du peuplement de maïs est largement déterminée par l'hétérogénéité apparue lors de la phase d'implantation (Pommel et Fleu$r y, 1989)$. Cette variabilité des stades jeunes reflète essentiellement les différences individuelles quant à l'importance des réserves de la graine (Abdel Rahman et Bourdu, 1986), la cinétique de leur imbibition (Boiffin et al, 1983; Blacklow, 1972), leur positionnement en profondeur dans le lit de semence (Alessi et Power, 1971).

Ces facteurs sont susceptibles de modifier, selon les cas, soit la durée des phases semisgermination et germination-levée, soit la vitesse de croissance au cours de cette dernière et de la phase levée-autotrophie. L'agencement de paramètres tels que vitesses de croissance, vitesses de développement, positionnement dans le temps de ces évolutions peut être complexe : grosse semence lente à germer, puis rapide à lever...

Or, il est tout à fait envisageable que les conditions de fonctionnement de la jeune plante munie d'un appareil photosynthétique efficace ne dépendent pas seulement de l'êtat final de la plantule à l'issue de la phase hétérotrophe, mais surtout de la façon dont cette situation a été atteinte. En somme, une caractérisation convenable de l'état de la plantule en fin de phase hétérotrophe demanderait un diagnostic de son état initial, c'est-à-dire poids de la semence d'origine et âge physiologique, donc, date de germination.

Comme l'utilisation des réserves constitue, lors des stades jeunes, la source énergétique presque exclusive, on peut chercher à estimer le poids initial de la semence en se fondant sur l'état de croissance de la plantule et l'état des réserves encore existantes en un instant donné. On écrira, pour cela, que le poids total de la plantule additionné des pertes par respiration est à tout moment égal à la diminution de poids sec de la semence depuis l'origine :

$$
\begin{gathered}
[G](1-H)-G R]=(E+R)+P \\
\text { ou : } E+R=[G](1-H)-G R]-P
\end{gathered}
$$

expression dans laquelle :

$E=$ poids sec de l'épicotyle, partie de la plantule située au-dessus du nœud scutellaire;

$R$ : poids sec des racines séminales;

$G I=$ poids frais de la graine au semis, de teneur en eau $H$ (quantité d'eau rapportée au poids frais);
$G R=$ poids sec de la graine au moment de la réolte de la plantule;

$P=$ perte de matière par respiration.

Durant la courte période qui nous intéresse, on pourrait supposer que la respiration est ellemême proportionnelle à la décroissance des réserves de la semence. On obtiendrait alors une relation linéaire entre la croissance de la plantule et la disparition des réserves de la semence:

$$
E+R=k[G I(1-H)-G R]-\mathrm{c}
$$

La constante $c$ exprime le fait que la mobilisation des réserves commence dès l'imbibition de la graine, alors que la croissance ne débutera qu'après la germination.

Nous allons donc chercher à établir les paramètres de ce modèle en observant toutefois qu'il ne pourra pas être utilisé sous cette forme pour diagnostiquer au champ les conditions d'origine de chaque plantule, puisque les variables $R$ et $G /$ n'y sont pas commodément accessibles.

Aussi cherchera-t-on :

- d'une part si $E$ peut être un bon estimateur du poids de la plantule $(E+R)$;

- d'autre part, à suivre l'évolution des réserves entre 2 dates $t 1$ et $t 2$, selon l'équation :

$$
\left[G I(1-H)-G R_{2}\right]-\left[G l(1-H)-G R_{1}\right]=G R_{1}-G R_{2}
$$

Si l'apparition de nouveaux organes foliaires ou racinaires n'obéit pas à la même dépendance linéaire que la croissance vis-à-vis de l'évolution des réserves, il en résultera des différences individuelles d'agencement entre la croissance et le développement, que nous chercherons à utiliser pour la caractérisation de la plantule.

Le modèle cesse d'être utilisable au démarrage d'une photosynthèse efficace, d'où découle la nécessité de situer le passage à l'autotrophie dans le temps et par rapport aux différentes variables observées sur la plante.

\section{MATÉRIEL ET MÉTHODES}

Les expérimentations ont été réalisées sous serre; les graines pesées étaient semées individuellement dans des godets remplis de vermiculite, puis irrigués jusqu'à écoulement; l'arrosage était ensuite renouvelé tous les 2 jours, ce qui permet de maintenir les plantules dans d'excellentes conditions hydriques. L'utilisation de la vermiculite permet une bonne imbibition et une bonne aération des semences; on peut espérer obtenir ainsi 
la simultanéité des germinations et une homogénéité des conditions de croissance, ce qui limitera la variabilité résiduelle.

La teneur en eau des graines est indépendante de leurs poids et présente des variations assez faibles au sein du même lot (cœefficient de variation autour de 5$8 \%$ ). Elle se situe, dans nos conditions de conservation, à $9,5 \pm 1 \%$ de la matière fraîche. Elle varie selon l'humidité atmosphérique ambiante (Blacklow, 1972; Bruckler, 1979). A partir de leur poids frais, on calcule le poids sec des graines semées par mesure de la teneur en eau d'une fraction aliquote de graines du même lot. La température du substrat est mesurée, $h$ par $\mathrm{h}$, à $2 \mathrm{~cm}$ de profondeur dans 6 godets répartis aléatoirement, avec des sondes à thermocouple $\mathrm{K}$ (chrome-nickel). Le cœefficient de variation étant $<3 \%$, nous avons utilisé la moyenne des 6 valeurs pour calculer les moyennes journalières; celles-ci sont ensuite additionnées quotidiennement, ce qui permet d'évaluer le temps physiologique des plantules en sommes de température (degrés.jour, base 0 ; toutes les températures étant supérieures à $10^{\circ} \mathrm{C}$, la conversion en une autre base est aisée). La répartition spatiale des godets sur la table de la serre était faite au hasard, de même que le choix des individus à récolter à l'intérieur de chaque traitement.

\section{Première expérimentation}

On étudie l'effet combiné du poids de la semence et de la profondeur. Les graines issues d'un lot de la variété $D E A$ et classées en petites ( $P G=140-200 \mathrm{mg}$ ) et grosses $(G G=310-370 \mathrm{mg})$ sont semées superficiellement $(S U=2 \mathrm{~cm})$ ou profondément $(P R=7 \mathrm{~cm})$. On effectue du 6 au $20^{\circ} \mathrm{j}$ après semis 10 récoltes, chacune comportant 15 plantules par traitement.

\section{Deuxième expérimentation}

Dans le but de mieux cerner l'effet des poids de chaque semence (GI), on a créé une gamme continue de valeurs : 11 classes de poids, d'effectifs et d'amplitude égaux (15 mg) ont été retenues de 170 à $335 \mathrm{mg}$. Les graines appartenant à la même variété que précédemment, sont semées à la profondeur intermédiaire de 5 $\mathrm{cm}$. Du $4^{\theta}$ au $12^{e}$ j suivant le semis, sont effectuées 7 récoltes d'une plantule par classe.

\section{Troisième expérimentation}

Le but est d'explorer un éventuel effet du génotype : les graines pesant de $260-305 \mathrm{mg}$, classe bien représentée dans chaque lot, et semées à $5 \mathrm{~cm}$ de profondeur comme dans l'expérimentation précédente, provenaient des génotypes $F_{7} \times F_{2}$, Monarque, Mohican, DEA. On a effectué une récolte quotidienne de 3 plantules par génotype, du $4^{\theta}$ au $14^{e} \mathrm{j}$ après le semis.
Les observations effectuées à chaque récolte sont les suivantes : poids sec de la semence; poids des parties de la plantule situées au-dessus de la semence (que nous appellerons par simplification épicotyle (E)); poids des racines séminales $(R)$; longueur du mésocotyle, c'est-à-dire du $1^{\text {er }}$ entrenœud entre le nœud scutellaire et le nœud coléoptilaire; plantule levée ou non, stades racinaires : apparition de la radicelle, apparition des racines latérales, apparition de ramifications sur celle-la ou celles-ci; apparition de racines nodales; stades foliaires : nombre de feuilles visibles hors du cornet foliaire, et nombre de feuilles adultes, présentant une ligule visible à la base.

L'étude statistique est fondée sur la méthode de l'analyse de la variance, les répétitions étant réparties au hasard. Les résultats des expérimentations 2 et 3 ont pour but de compléter ceux de la première, qui est la principale; dans la suite du texte, et par commodité d'écriture, nous ne spécifierons le numéro de l'expérimentation que lorsqu'il s'agira des 2 dernières.

\section{RÉSULTATS}

\section{Croissance et développement de la plantule selon la taille de la semence}

\section{Modélisation de la croissance en fonction de la mobilisation des réserves}

La dépendance linéaire de la croissance vis-àvis de la disparition des réserves s'exprime selon la relation (1).

En fait, la croissance ne débute qu'après la germination. Or, des expériences préliminaires nous ont montré que la fraction des réserves mobilisées pour la germination était constante à l'intérieur d'une variété donnée : pour l'hybride DEA, nous avons obtenu, sur 53 cas, la valeur moyenne de $2,2 \%$ du poids frais.

Le poids sec de la semence en germination s'écrit donc, compte tenu de la teneur en eau originelle de $10,4 \%$ :

$$
G I=(1-0,104-0,022)
$$

En replaçant $G /$ par cette valeur dans (1), on annule la constante $c$ :

$$
E+R=k^{\prime}(0,874 G I-G)
$$

La figure 1 nous montre que la croissance dépend linéairement de la disparition des réserves, jusqu'à ce que la plantule pèse $76 \mathrm{mg}$ dans le cas des petites semences, et $130 \mathrm{mg}$ dans celui des grosses. On trouvera sur la figure 2 un 


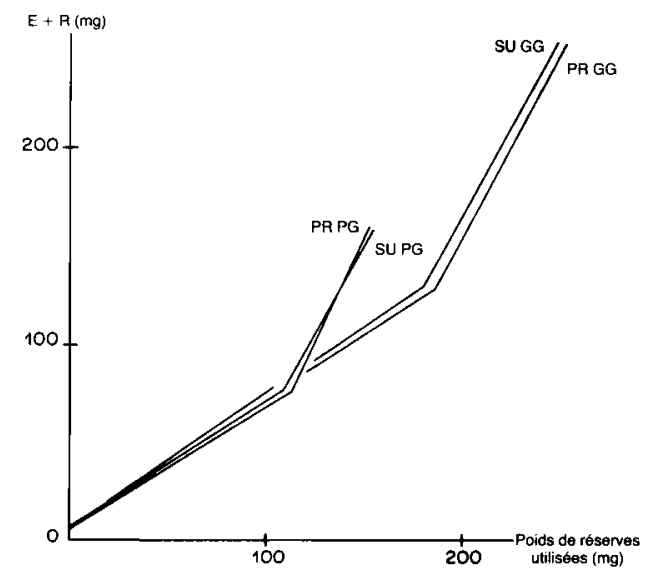

Fig 1. Croissance des plantules en fonction de la diminution des réserves de la graine postérieurement à la germination, et selon le traitement. $E$ : poids sec de l'épicotyle; $R$ : poids sec des racines; semis profond (PR) ou superficiel (SU); graines petites $(P G)$ ou grosses $(G G)$.

exemple de la dispersion des valeurs. On obtient, par le calcul, les équations suivantes (en $\mathrm{mg})$ :

\section{Traitement SUPG :}

$$
\begin{aligned}
& E+R=0,650(0,874 G l-G R)+6,4 \\
& \text { avec } r=0,99
\end{aligned}
$$

\section{Traitement PRPG :}

$$
\begin{aligned}
& E+R=0,617(0,874 \mathrm{Gl}-\mathrm{GR})+7,6 \\
& \text { avec } r=0,99
\end{aligned}
$$

\section{Traitement SUGG :}

$$
\begin{aligned}
& E+R=0,672(0,874 \mathrm{Gl}-\mathrm{GR})+7,5 \\
& \text { avec } r=0,98
\end{aligned}
$$

\section{Traitement PRGG :}

$$
\begin{aligned}
& E+R=0,656(0,874 G I-G R)+5,9 \\
& \text { avec } r=0,98
\end{aligned}
$$

On note que la linéarité est élevée, et que les pentes - quoique différentes - sont voisines dans chacune des situations (cœefficient de variation de $3,6 \%$ ).

Bien que faibles, les résidus sont non nuls, ce qui pourrait n'être que la conséquence d'une certaine imprécision de l'estimation de la teneur en eau des graines semées.

A partir de la mise en commun des résultats des 4 traitements, on conserve encore une étroite linéarité. En effet, pour $E+R<76 \mathrm{mg}$, on obtient :

$$
\begin{aligned}
& E+R=0,606(0,874 G I-G R)+8,2 \\
& \text { avec } r=0,98
\end{aligned}
$$

De façon analogue pour la deuxième expérimentation, qui se déroule entièrement pendant la phase hétérotrophe (fig 2), on calcule la relation :

$$
\begin{aligned}
& E+R=0,658(0,874 G l-G R)+1,1 \\
& \text { avec } r=0,99
\end{aligned}
$$

La pente se situe dans la gamme de variation des précédentes, et le résidu est très faible.

Entre les 2 catégories de taille, l'écart relatif des pourcentages de réserves mobilisées à une date donnée, bien que très hautement significatif, n'est que de $7,3 \%$ en moyenne (tableau I), à l'avantage des petites graines, alors que l'écart relatif des poids de semences est de $61 \%$.

La quantité absolue des réserves utilisées pendant une période donnée est donc beaucoup plus élevée chez les plantules issues de grosses semences, et la dépendance de la croissance vis-à-vis de ce paramètre se traduit par une croissance nettement plus rapide de ces dernières (fig 3), ce qui confirme les résultats d'Abdel Rahman et Bourdu (1986).

La supériorité des petites semences pour le taux d'utilisation des réserves à une date donnée, très hautement significative, se combine avec la moindre consommation de réserves avant germination en valeur absolue pour engendrer une croissance ramenée au poids initial de la semence, qui est, elle aussi, plus élevée de façon très hautement significative (tableau II).

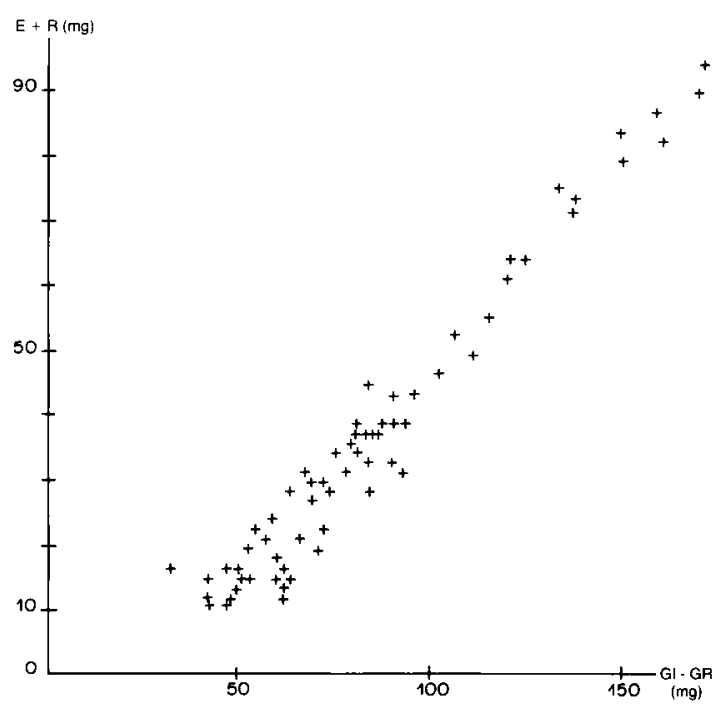

Fig 2. Croissance des plantules en fonction de la diminution des réserves de la graine ( $2^{\circ}$ expérimentation). Gl: poids initial de la semence; $G R$ : poids de la semence à la récolte. 
Tableau I. Pourcentage de réserves utilisées [(GIGR)/GR] × 100, selon la date (degrés.jour) et le traitement. Analyse de la variance : Probabilité : $5 \%$; Effet profondeur : THS; Effet taille de graine : THS; Interaction : S; Cœfficient de variation de l'erreur : $6,7 \% ; S$ : significatif; THS : Très hautement significatif.Classement (Newman-Keuls à 5\%): $P R(P G): A ; S U(P G): B ; P R(G G): B ; S U(G G): C ; P R: A ; S U: B ; P G: A ; G G$ : B

\begin{tabular}{lllllllllll}
\hline$d^{\circ} x j$ & 91 & 124 & 158 & 206 & 223 & 239 & 256 & 273 & 291 & 330 \\
\hline SU(PG) & 11 & 18,8 & 31,8 & 45,2 & 53,2 & 57,4 & 68 & 67 & 74,6 & 84,4 \\
SU(GG) & 09,8 & 18,8 & 28,6 & 45 & 50 & 53,2 & 60,6 & 66 & 69,2 & 80,6 \\
& & & & & & & & & & \\
$P R(P G)$ & 09 & 23,2 & 35,4 & 55 & 55,8 & 62,6 & 70,2 & 75,4 & 81,2 & 85,2 \\
$P R(G G)$ & 11,4 & 20,6 & 29,4 & 46,8 & 50,4 & 54,6 & 64,2 & 68,4 & 73,2 & 82,8 \\
\hline
\end{tabular}

En résumé, nous obtenons donc une croissance nettement plus rapide des plantules issues de grosses semences, mais une efficience légèrement supérieure des plantules issues de petites semences, dans l'utilisation des réserves de la graine.

\section{Date d'acquisition des stades physiologiques}

Le tableau III représente les dates d'apparition des feuilles, de leur caractère adulte, et d'apparition de ramifications sur la radicelle ou les racines latérales.

Comme l'a observé Hébert (1986), le rythme d'apparition des feuilles, très élevé au départ, se ralentit à la $4^{e}$ feuille, et le passage des premières feuilles à l'état adulte est plus lent.

On voit qu'il n'existe pas de différence systématique et importante entre plantules issues de graines des 2 tailles. On note également une correspondance régulière des dates entre stades foliaires et racinaires : les ramifications sur la radicelle apparaissent en même temps que la $2^{\theta}$ feuille, ou juste un peu avant. Les ramifications sur les racines latérales apparaissent en même temps que la $3^{\theta}$ feuille.

L'observation des stades foliaires ou racinaires permettra donc d'apprécier l'âge d'une plantule depuis sa germination, indépendamment de la connaissance de la taille de la semence d'origine. II est plus difficile de remonter jusqu'au semis : il faut, en effet, garder à l'esprit que la durée de la phase semis-germination - ici 40 degrés.jour - peut être allongée en cas de conditions d'imbibition défavorable (Boiffin et al, 1983).

\section{Acquisition de l'autotrophie}

La figure 1 montre que la relation linéaire entre la croissance et la mobilisation des réserves subit une rupture nette : celle-ci correspond à un pourcentage de réserves utilisées qui pourrait être indépendant de la taille de la semence, puisqu'il s'élève à $67 \%$ dans le cas des petites graines, et $63 \%$ dans celui des grosses.

Le changement de pente - qui se produit à 240 degrés.jour environ - s'accompagne chez les petites semences, ou est précédé de peu chez les grosses, d'une chute provisoire du poids des racines (tableau IV), ce qui permet à Bourdu et Grégory (1983) de parler de crise de transition : on peut penser que, pendant une brève période, l'entrée de flux carboné dans les racines est insuffisante pour compenser les pertes respiratoires.

On voit sur le tableau Ill que ce passage à l'autotrophie se produit après l'apparition de la $3^{\theta}$ feuille, l'obtention du caractère adulte de la 1 re feuille, et l'apparition de ramifications sur les racines latérales. II lui correspond une chute du $R G R$ (taux de croissance relatif), suivie de sa

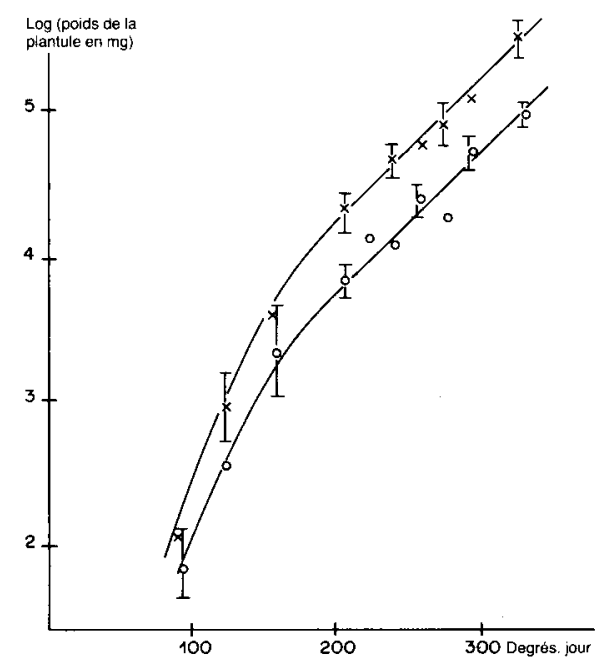

Fig 3. Evolution du logarithme du poids de plantule en fonction du temps (degrés-jour) (Exemple des semis superficiels). Intervalles de confiance à $5 \%$ I ; Grosses semences : $x$; Petites semences : 0 . 
Tableau II. Croissance de la plantule rapportée au poids initial de la semence $[(E+R) / G] \times 1000$ en fonction de la date (degrés.jour) et du traitement.

Analyse de la variance : Probabilité : 5\%; Effet profondeur : THS; Effet taille de graine : THS; Interaction : NS; Cœfficient de variation de l'erreur : 15,4; THS : Très hautement significatif : NS : Non significatif.

\begin{tabular}{lllllllllll}
\hline$d^{\circ} \times j$ & 91 & 124 & 158 & 206 & 223 & 239 & 256 & 273 & 291 & 330 \\
\hline SU(PG) & 33,6 & 68,4 & 153,8 & 241,2 & 372,8 & 332,6 & 446 & 420 & 601,2 & 768,4 \\
SU(GG) & 23,6 & 58,4 & 108,6 & 236,8 & 252,6 & 311,2 & 358,8 & 415,4 & 501 & 700,4 \\
& & & & & & & & & & \\
$P R(P G)$ & 39,2 & 97,2 & 184,6 & 297,4 & 360 & 370,2 & 432 & 499,6 & 692 & 790,8 \\
$P R(G G)$ & 25,6 & 63,8 & 123,4 & 234,4 & 236,6 & 310 & 393,6 & 446,4 & 561,4 & 753,4 \\
\hline
\end{tabular}

stabilisation, apparaissant sur la figure 3 comme la pente du logarithme de la croissance en fonction du temps.

Durant le début de la phase autotrophe, la photosynthèse constitue la source énergétique essentielle, à laquelle s'additionnent les réserves encore mobilisées. Sur la figure 1, la croissance est encore représentée par des tracés à allure linéaire, mais en prolongeant l'expérimentation, les réserves de la semence s'épuiseraient entièrement, et la pente tendrait vers l'infini.

\section{Effets de la profondeur de semis}

On voit sur le tableau II que la levée est plus précoce chez les plantes ayant subi un traitement superficiel, ce qui s'explique par le moindre allongement que doit subir la plantule pour atteindre la surface. Par la suite, à partir de l'apparition de la $2^{\theta}$ feuille, il n'y a plus de différence systématique de stade entre les 2 profondeurs de semis.

On sait que sitôt la levée, la lumière qui frappe l'extrémité du coléoptile est transmise au mésocotyle dont elle inhibe l'élongation (Mandoli et Briggs, 1982). Effectivement, dans les traitements «semis profond», la longueur moyenne finale des mésocotyles était de $26,4 \mathrm{~mm}(n=73$, $S=5,44)$ et seulement de $9,1 \mathrm{~mm}(n=78, S=$ 3,51 ) dans les traitements «semis superficiel», la différence est très hautement significative.

Cette longueur supérieure du premier entrenœud chez les semis profonds a pour conséquence une modification du rapport d'allométrie, au détriment des racines; toutefois, nous ne pou- vons en observer les conséquences ultérieures sur le système racinaire, vu le faible volume de sol à sa disposition. Par ailleurs, nous constatons (tableau I) que le taux d'utilisation des réserves est alors légèrement plus élevé, mais de façon très hautement significative : tout se passe comme si les besoins plus ou moins intenses du puits mésocotyle contrôlaient la vitesse d'utilisation de la source semence.

Cette utilisation plus rapide des réserves engendre un taux de croissance légèrement supérieur, mais de façon très hautement significative, des plantules semées en profondeur (tableau II).

En conséquence, les plantules semées profondément atteindront un stade donné, en ayant utilisé une fraction supérieure de leurs réserves, et en montrant une croissance supérieure.

\section{Utilisation du modèle de croissance pour la caractérisation de la plantule au champ}

Nous avons construit un modèle reliant linéairement la croissance totale de la plantule à la quantité de réserves utilisées. Cependant, il n'est pas possible d'accéder rapidement, dans les conditions naturelles, à une estimation non biajsée du poids des racines, en raison de l'adhérence des particules terreuses. Nous avons donc voulu apprécier la valeur du poids de l'épicotyle seul comme indice du poids total de la plantule, en calculant la corrélation existant entre les 2 variables. Cette recherche a été faite séparément pour les 2 types de semis, en vue de tenir compte de la variation d'allométrie qu'introduit la différence de profondeur de semis. 


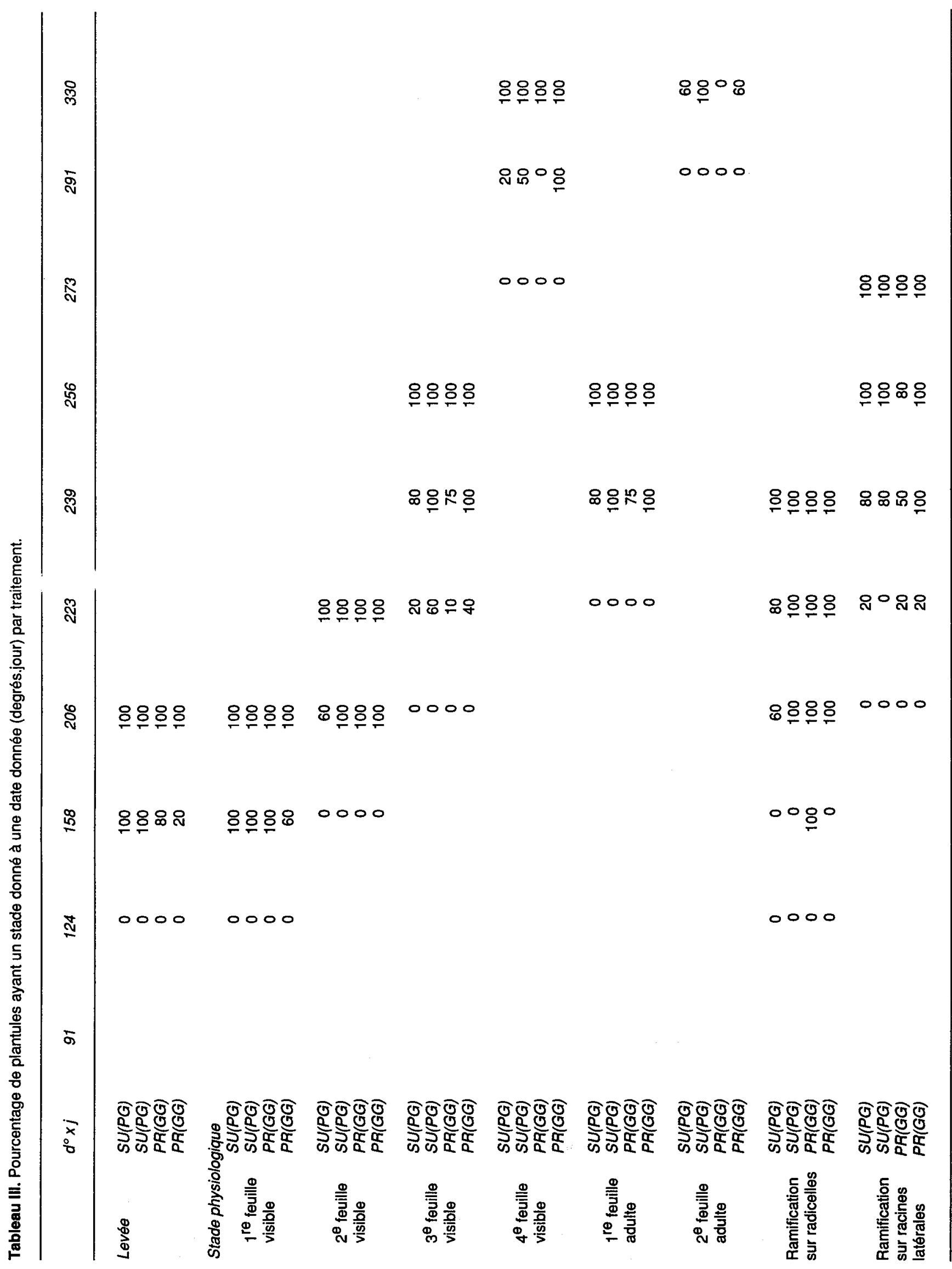


Tableau IV. Vitesse de croissance racinaire $\left(\mathrm{mg} \times\right.$ degrés.j $^{-1} \times 100$ ) entre la date «i-1» (degrés.jour) et la date «i».

\begin{tabular}{lrrrrrrr}
\hline$d^{\circ} x j$ & 124 & 158 & 206 & 223 & 239 & 256 & 273 \\
\hline$S U(P G)$ & 17,1 & 24,6 & 18,5 & 38,8 & $-21,2$ & 62,3 & $-39,4$ \\
$S U(G G)$ & 22,6 & 27,1 & 42,5 & $-7,6$ & 106,0 & 16,5 & 37,1 \\
$P R(P G)$ & 16,5 & 15,8 & 13,1 & 36,5 & $-10,0$ & 24,7 & 30,0 \\
$P R(G G)$ & 22,3 & 28,2 & 40,0 & $-35,9$ & 60,6 & 100,0 & 26,5 \\
\hline
\end{tabular}

Semis superficiels (SU)

$$
E=0,61(E+R)-6,6 \text {, avec } r=0,987
$$

\section{Semis profonds (PR)}

$$
E=0,628(E+R)-3,8 \text {, avec } r=0,991
$$

La corrélation linéaire entre $E$ et $(E+R)$ est très étroite dans chaque cas, et la variable $E$ peut donc être valablement utilisée pour représenter la plantule entière.

En partant d'un traitement commun des résultats des 2 profondeurs, on obtient entre $(E+R)$ et $E$ la relation :

$$
(E+R)=(E+5,2) / 0,619
$$

et, en effectuant un changement de variable dans l'expression (2):

$$
(E+5,2) / 0,619=0,606(0,874 G I-G R)+8,2
$$

soit :

$$
E=-0,375 G R+0,328 G I-0,124
$$

Dans une population de plantules en croissance hétérotrophe, la croissance individuelle des épicotyles en fonction des poids concomitants de semences est représentée par une famille de droites parallèles issues de l'axe des $x$ en des points d'abscisse 0,87 Gl. Autrement dit, à partir de la mesure de $E$ et de $G R$ sur une plantule quelconque, à n'importe quel moment de la croissance hétérotrophe, on peut calculer Gl.

De la même façon, dans la deuxième expérimentation, le poids de l'épicotyle est linéairement lié au poids total de la plantule par une relation voisine :

$$
E=0,605(E+R)-3,576, \text { avec } r=0,98
$$

et, en effectuant le même changement de variable dans la relation (3), on obtient :

$$
E=0,398 G R+0,348 \mathrm{Gl}-2,46
$$

relation très voisine de la précédente.

\section{Effets propres au lot de semence}

Nos essais en vases de végétation ont été effectués à partir de lots de semence ayant une bonne valeur germinative. Si l'on se trouvait au champ devant un peuplement provenant d'un lot de semence médiocre, l'appréciation de l'âge de la plantule serait biaisée car, dans ce cas, les semences utilisent moins rapidement leurs réserves (Abdul-Hamid, 1986).

Dans le même ordre d'idées, une extrapolation directe de la méthode aux divers génotypes n'est pas possible car, au cours de la croissance hétérotrophe, Monarque utilise ses réserves plus rapidement que DEA, $F_{7} \times F_{2}$ ou Mohican (tableau $\mathrm{V})$, ceci de façon significative.

Cette caractéristique se traduit par une vitesse de croissance plus rapide pour Monarque pendant la phase hétérotrophe, que pour les autres hybrides cités.

\section{DISCUSSION - CONCLUSION}

Le poids d'une plantule en phase hétérotrophe est directement proportionnel à la quantité de réserves mobilisées depuis la germination, pour toute taille de graine et profondeur de semis. Comme la vitesse relative d'utilisation des réserves varie dans des proportions bien moindres que les poids de semence d'origine, les plantules issues de grosses semences mobilisent en un temps donné une quantité de réserves bien supérieure, et connaissent donc une croissance plus rapide que celles issues de petites semences. La vitesse de développement n'est toutefois pas modifiée. 
Tableau V. Evolution du pourcentage de réserves utilisées en fonction du temps, chez 4 génotypes de maïs.

Analyse de la variance : Génotype : Très hautement significatif; Interaction génotype x temps : Significatif 10,03\%; Ccefficient de variation de l'erreur : $6,9 \%$

\begin{tabular}{lllllllllllll}
\hline Degrés-jour & 97 & 119 & 142 & 165 & 189 & 213 & 238 & 264 & 289 & 309 & $\begin{array}{c}\text { Test de } \\
\text { Newman-Keuls (*) }\end{array}$ \\
\hline
\end{tabular}

Génotype :

\begin{tabular}{|c|c|c|c|c|c|c|c|c|c|c|}
\hline \multirow{4}{*}{$\begin{array}{l}\text { Monarque } \\
\text { DEA } \\
F_{2} \times F_{7} \\
\text { Mohican }\end{array}$} & 28,2 & 38,2 & 49 & 57 & 72 & 78,2 & 85,7 & 90,2 & 88,5 & 90 \\
\hline & 29,7 & 33,7 & 41,5 & 50 & 61,5 & 69,7 & 77,2 & 80,5 & 87 & 88,5 \\
\hline & 24,7 & 31,2 & 41,2 & 47 & 59,7 & 69,2 & 77,5 & 80,2 & 75 & 86,2 \\
\hline & 21,7 & 31,2 & 35,7 & 44,2 & 52,7 & 70,7 & 68 & 74,7 & 79,5 & 82 \\
\hline
\end{tabular}

La date de passage à l'autotrophie dépendant étroitement de la fraction des réserves mobilisées et du stade physiologique atteint; les plantules issues de grosses semences démarrent leur période photosynthétique avec un poids d'organes chlorophylliens supérieur : ceci pourrait avoir d'importantes conséquences pour le déroulement de la phase de croissance exponentielle, dont l'état initial se trouve ainsi modifié favorablement.

Chez les petites graines, les réserves encore disponibles au moment du passage à l'autotrophie sont en moindre quantité. Or celles-ci constituent une marge de sécurité : les plantules issues de petites semences montreront une plus grande fragilité vis-à-vis de conditions d'environnement défavorables telles que obstacles structuraux, périodes sombres, et surtout températures basses puisque Derieux et al (1989) ont montré que le démarrage de la photosynthèse était plus coûteux en réserves dans des conditions froides. On pourrait même arriver à ce que l'implantation soit rendue impossible.

La connaissance du poids de la semence d'origine, qui détermine la capacité de résistance du végétal à des conditions adverses, constitue donc un élément essentiel de la caractérisation d'une plantule.

Le modèle que nous avons construit dans ce but a été mis en paramètres au cours de 2 essais, en conditions de substrat homogène. Les relations obtenues sont les suivantes :

$$
E=-0,375 G R+0,328 G I-0,124(4)
$$

dans le premier cas, et :

$$
E=-0,398 G R+0,348 G I-2,46(5)
$$

dans le second.
Rappelons que $E$ représente le poids de l'épicotyle, partie de la plantule située au-dessus du nœud scutellaire; $G l$, le poids frais de la graine au semis, et $G R$ le poids sec de la graine au moment de la récolte de la plantule.

On voit que la pente liant les 2 variables $E$ et $G R$ est indépendante du poids initial de semence Gl, qui modifie seulement le terme constant. On peut donc rechercher empiriquement de telles relations dans les conditions naturelles, où le poids des semences est variable et inconnu.

Dans 2 situations au champ, des pesées effectuées sur un grand nombre de plantules nous ont donné les relations suivantes:

$$
E=-0,415 G R+93,9(6) ; n=215 \text { individus }
$$

et

$$
E=-0,463 G R+100,5(7) ; n=180 \text { individus }
$$

Le cœefficient de variation sur les pentes des relations (4)-(7) est de $9 \%$, et il importe d'obtenir des résultats complémentaires permettant de décider s'il s'agit d'une variation résiduelle, ou si elle est sous la dépendance de certains paramètres à déterminer. Une fois ce point élucidé, on pourra donc espérer apprécier le poids originel de la graine de n'importe quelle plantule en croissance hétérotrophe à partir du poids de son épicotyle et du poids concomitant de la semence.

L'ampleur des réserves de la semence n'agissant pas sur la vitesse du développement modifiera l'agencement de celui-ci avec la croissance.

Concrètement, entre des plantules présentant le même poids d'épicotyle, celles qui auront un poids concomitant de semence plus élevé et présenteront un retard de stade, seront issues 
de plus grosses semences, germées plus tard et ayant utilisé une fraction moindre de leurs réserves.

Entre des plantules de même stade, celles qui présenteront un épicotyle plus lourd et un poids concomitant de semence plus élevé seront issues de plus grosses graines, germées à la même date et ayant mobilisé une fraction des réserves légèrement inférieure, mais une quantité totale très supérieure.

Un accroissement de la profondeur de semis génère des modifications de la phase d'allongement du mésocotyle : la disparition des réserves et la croissance sont plus rapides, mais la survenue de la levée, qui est retardée, requiert la mobilisation d'une fraction supérieure des réserves.

Cette tardivité peut être encore accrue par certaines conditions du milieu, comme une structure motteuse, qui accroît la tortuosité du mésocotyle (Duparque et al, 1988), ou une croûte de battance. La marge de sécurité que constitue l'existence de réserves encore disponibles lors de la levée sera réduite, et ce handicap se combinera avec la faible ampleur des réserves au départ : ainsi, quand la levée vient de se terminer, les petites graines semées profondément ne disposent plus que de $45 \%$ de leurs réserves (soit $75 \mathrm{mg}$ ) contre $71 \%(235 \mathrm{mg}$ ) pour les grosses graines semées superficiellement.

\section{REMERCIEMENTS}

L'auteur tient à remercier M Sébillotte pour la contribution très positive qu'il a apportée dans la discussion des résultats de cette étude.

\section{RÉFÉRENCES}

Abdel Rahman N, Bourdu R (1986) Effet de la taille et de la forme des grains sur quelques caractéristi- ques du développement du maïs au stade jeune. Agronomie 6, 181-186

Abdul-Hamid I (1986) Influence de la qualité semencière sur la germination, la croissance hétérotrophe et le devenir des plantules de maïs. Thèse Doc Univ Rennes I, $148 \mathrm{p}$

Alessi J, Power JF (1971) Corn emergence in relation to soil temperature and seedling depth. Agron J 63, 717-719

Blacklow WM (1972) Mathematical description of the influence of temperature and seed quality on imbition by seeds of corn (Zea mays L). Crop Sci 12, 643-646

Boiffin J, Bruckler L, Aubry C (1983) Rôle des propriétés physiques du lit de semence sur l'imbibition et la germination. III. Valeur prévisionnelle d'un modèle d'imbibition au champ et caractérisation des lits de semences. Agronomie 3, 291-302

Bourdu R, Gregory N (1983) Etude comparée du début de la croissance chez divers génotypes de maïs. Agronomie 3, 761-770

Bruckler $L$ (1979) L'imbibition et la germination des semences en conditions sèches : influence des caractères physiques du lit de semence. Th DDI, INAPG, Paris, $119 p$

Derieux M, Bourdu R, Duburcq JB, Boizard H (1989) La crise de croissance de la plantule de maïs à basse température. Agronomie 9, 207-212

Duparque A, Fleury A, Bloc D (1988) Contribution à l'étude au champ de la croissance juvénile d'un peuplement de maïs (Zea mays $\mathrm{L}$ ). Mémoire Univ, Paris Sud, $43 p$

Hébert $Y$ (1986) Expression de la variabilité génétique du phénomène d'hétérosis au cours de la vie de la plante chez le maïs. Thèse Doct Ing, ENSA Rennes, $142 \mathrm{p}$

Mandoli DF, Briggs WR (1982) The photoperceptive sites and the function of tissue light-piping in photomorphogenesis of etiolated oat seedlings. Plant Cell Environ 5, 137-145

Pommel B, Fleury A (1989) Etude de la variabilité individuelle chez le maĩs à l'issue de la phase d'implantation. Conséquences sur la croissance et la production de grain. Agronomie 9, 467-475 